\title{
Spitzer Infrared Array Camera (IRAC) Pipeline: Final Modifications and Lessons Learned
}

\author{
Patrick J. Lowrance ${ }^{\mathrm{a}}$, Sean J. Carey ${ }^{\mathrm{a}}$, Jason A. Surace ${ }^{\mathrm{a}}$, James G. Ingalls ${ }^{\mathrm{a}}$, Willam Glaccuum ${ }^{\mathrm{a}}$, \\ Jessica E. Krick ${ }^{\mathrm{a}}$, John Stauffer ${ }^{\mathrm{a}}$ \\ ${ }^{a}$ Spitzer Science Center, 1200 East California Blvd, Pasadena, CA, USA 91125
}

\begin{abstract}
In more than ten years of operations, the Spitzer Space Telescope has conducted a wide range of investigations from observing nearby asteroids to probing atmospheric properties of exoplanets to measuring masses of the most distance galaxies. Observations using the Infrared Array Camera (IRAC) at 3.6 and 4.5um will continue through mid-2019 when the James Webb Space Telescope will succeed Spitzer. In anticipation of the eventual end of the mission, the basic calibrated data reduction pipeline designed to produce flux-calibrated images has been finalized and used to reprocess all the data taken during the Spitzer warm mission. We discuss all final modifications made to the pipeline.
\end{abstract}

Keywords: infrared, pipeline, image processing, distortion, calibration

\section{INTRODUCTION}

The two InSb arrays of Infrared Array Camera ${ }^{1,2}$ (IRAC) aboard Spitzer Space Telescope ${ }^{3}$ are currently operating successfully into the seventh year past the original $\sim 5.5$ year cryogenic mission. Prior to the end of the cryogenic mission, it was noted that the passive cooling would continue to achieve telescope and multiple instrument chamber (MIC) temperatures of $\sim 30 \mathrm{~K}$. The thermal background of the telescope and the noise properties ${ }^{4}$ of the two InSb arrays of IRAC for passively cooled Spitzer provide comparable sensitivity ${ }^{5}$ to the cryogenic observations at 3.6 and $4.5 \mu \mathrm{m}$ during the post-cryogenic mission, i.e. the "warm mission".

\section{SYSTEMATIC CHANGES TO PROCESSING}

The Basic Calibrated Data (BCD) reduction pipeline has not changed extensively since the first years of the Spitzer mission. The backbone of the pipeline consists of stand-alone modules for each step (Figure 1). These are each described in greater detail in the IRAC data handbook ${ }^{6}$. There have been systematic changes made to enhance the basic data that include tracking latents, improving the distortion correction, and pointing refinement. Each is discussed below in detail.

\subsection{Flagging latents}

Residual images of a source can be found on the array after a bright source has been moved off a pixel. These persistent images can be mistaken for faint sources and be an additional source of noise in photometry. The "latent" images are produced when a small fraction of the photoelectrons are trapped after illumination of the pixel. They often begin as positive flux remaining after a bright source has been observed, but can turn negative, releasing a "hole" rather than the trapped electrons.

Two differences were found in the warm era of IRAC operations. The traps have characteristic decay rates, which were found to be hours to days as opposed to less than an hour observed in the cryogenic mission. They were also found to be formed from stars in a lower brightness range than in the cryogen era, meaning they became much more pervasive. The earlier pipeline had flagged possible latents using a flux threshold and a decay rate within an observation, as the data reduction pipeline had been developed around an observation-centered approach. Due to longer decay rates and lower flux thresholds, a new multi-observation approach was needed for flagging latents. The break between downlinks was chosen and is now used as a reasonable "set" of observations.

After the data is processed through the pipeline, all observations between two downlinks are examined for the stars which hit the threshold. That pixel is then flagged in a table for the characteristic decay time. For many observations, this

Space Telescopes and Instrumentation 2016: Optical, Infrared, and Millimeter Wave, edited by

Howard A. MacEwen, Giovanni G. Fazio, Makenzie Lystrup, Proc. of SPIE Vol. 9904,

$99045 Z$ · C 2016 SPIE · CCC code: 0277-786X/16/\$18 · doi: 10.1117/12.2233804

Proc. of SPIE Vol. $990499045 Z-1$ 
can be multiple stars. Once this master list is created, all observations within that set are reprocessed and the masks are updated from the latent table created. This was a robust method to flag latents across multiple observations.

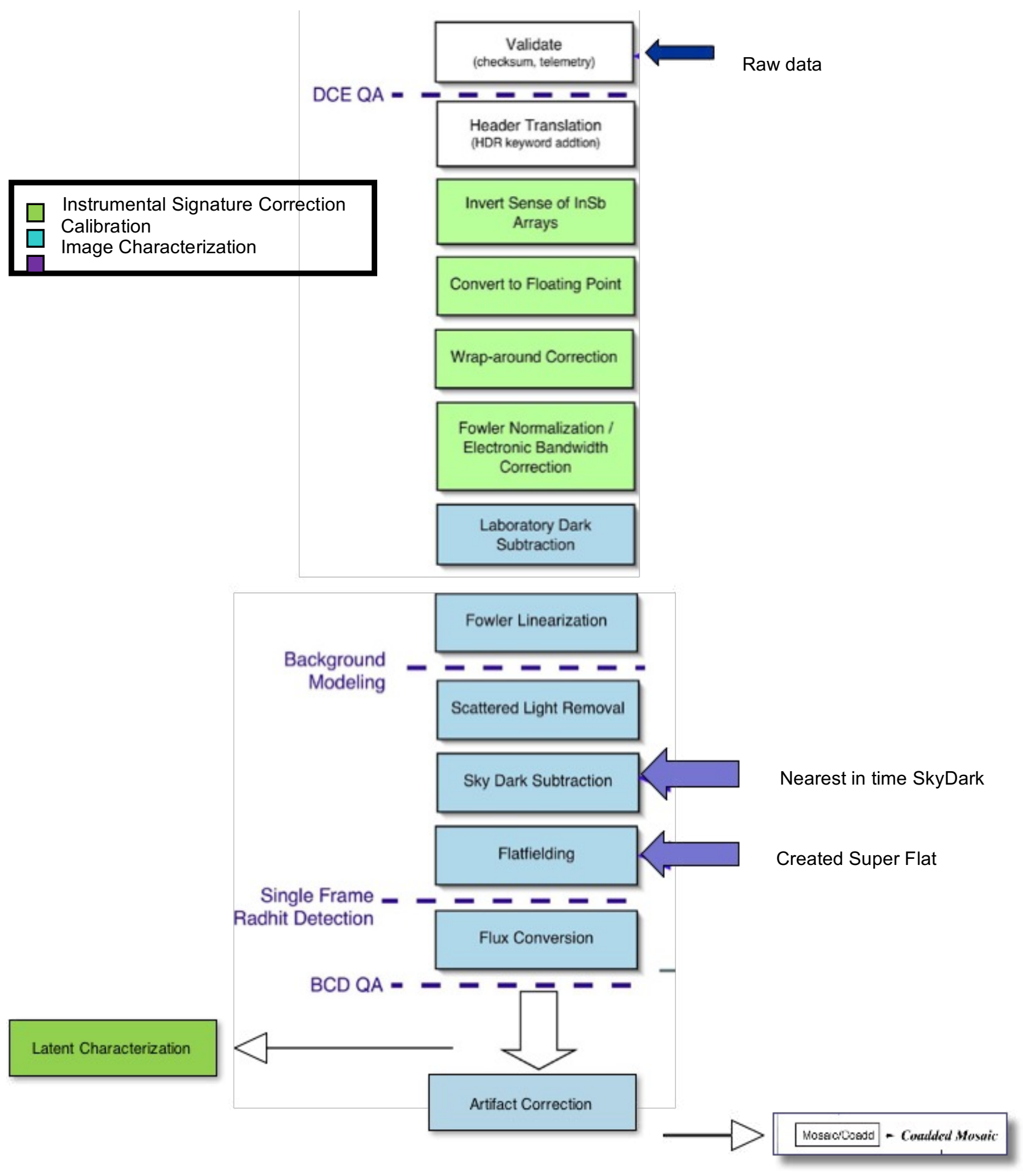

Figure 1. Data flow of the processing of raw data within the basic calibrated data pipeline for IRAC camera on Spitzer. 


\subsection{Updated Distortion Correction}

For most of the Spitzer mission, a derived third order polynomial distortion correction with a residual error of $\sim 100$ mas, or $<0.1$ pixel, exceeded the needs for most science observations. A driver to increase the correction to provide better measurements included the discoveries of brown dwarfs within the solar neighborhood. The recent scientific mission of $\mathrm{Gaia}^{7}$ to measure distances to stars will not be able to reach these faintest neighbors that represent the lower limit of the star formation mass distribution. Recent all-sky surveys continue to reveal more nearby brown dwarfs ${ }^{8}$, and Spitzer provides a unique opportunity.

An accurate distance measurement of the parallax of these objects is needed to fully analyze the apparent luminosity and relate it to the mass function. The average distance of $20 \mathrm{pc}$ requires a noise floor $<15 \%$ of $50 \mathrm{mas}$, or 7.5 mas. These objects are exceedingly dim to target with most ground based observatories and the fainter ones would take many years of dedicated time on larger 8 - to 10-m class telescopes.

\subsubsection{Current Convention}

The Simple Imaging Polynomial (SIP) convention ${ }^{9}$ was developed early in the cryogenic mission to provide a means for representing a non-linear geometric distortion with a polynomial within the FITS header keywords. As defined, $u, v$ are relative pixel coordinates with origin at CRPIX1, CRPIX2 and $x, y$ are "intermediate world coordinates" in degrees, with origin at CRVAL1, CRVAL2. Let $f(u, v)$ and $g(u, v)$ be the quadratic and higher- order terms of the distortion polynomial.

Then

$$
\left(\frac{x}{y}\right)=\left(\frac{C D 1 \_1}{C D 2 \_1} \frac{C D 1 \_2}{C D 2 \_2}\right)\left(\frac{u+f(u, v)}{v+g(u, v)}\right)
$$

We define $\mathrm{A} \_p \_q$ and $\mathrm{B} \_p \_q$ as the polynomial coefficients for polynomial terms $u u^{p} v^{q}$. Then

$$
\begin{array}{ll}
f(u, v)=\sum_{p, q} A_{-} p_{-} q u^{p} v^{q}, & p+q \leq A_{-} \text {ORDER }, \\
g(u, v)=\sum_{p, q} B_{-} p_{-} q u^{p} v^{q}, & p+q \leq B_{-} \text {ORDER }
\end{array}
$$

Currently, the distortion is represented by a third order polynomial. Using the equations above, it can be written as

$$
f(u, v)=A_{-} 2 \_0 u^{2}+A_{-} 0 \_2 v^{2}+A_{-} 1 \_1 u v+A_{-} 1 \_2 u v^{2}+A_{-} 2 \_1 u^{2} v+A_{-} 3 \_0 u^{3}+A_{-} 0 \_3
$$

where the coefficients are listed within the header of the FITS images. The CDijj keywords encode skew as well as rotation and scaling. The CD- matrix values together with the higher-order distortion polynomials define a unique transformation from pixel coordinates to the plane-of-projection. For Spitzer, we also provide polynomials for the reverse transformation, for fast inversion.

\subsubsection{Improvement of the Polynomial}

To improve the correction within the boundaries of the current polynomial correction, the order of the polynomial correction was increased from a third order to a fifth order. Working to approach a reduction in the measurement noise floor by $\sim 5 \mathrm{x}$, or $\sim 20 \mathrm{mas}$, the number of iterations of the observations could possibly approach the 5-10 mas needed for pushing the parallax measurable out to $20 \mathrm{pc}$.

The approach centered on known globular clusters, which is a customary method for deriving a distortion correction. Data sets were chosen from IRAC observations of clusters NGC 6791 and NGC 2420. Since no "truth" set of positions existed for the entire region covered by the IRAC observations, it was useful to attempt to create an astrometric catalog using the observations. As a test of this method, a catalog was created using the many observations NGC 6791. Eight mosaics were created from the data. As the $3^{\text {rd }}$ order distortion correction created an astrometry in a single image of better than 0.2 ", then a mosaic of many single images should be good to 0.1 " over most of the area. Therefore, a 
combination of centroids across these mosaics was expected to give much better. The catalog required the star to appear in at least three of the eight mosaics. A subset of the catalog of point source positions representing a smaller area of the region had HST measured astrometry ${ }^{10}$ that claimed accuracies better than 0.1 ", and a comparison revealed smaller and significantly better residuals in the IRAC derived catalog. A similar catalog was then created for NGC2420 and those point sources with large residuals and not found in several frames were systematically removed. With these "truth" catalogs residual offsets were measured for each source from the position in a single frame given by the $3^{\text {rd }}$ order and the catalog position, which represented how much error was unaccounted for in the $3^{\text {rd }}$ order polynomial. Similar residuals across the array were found in both clusters.

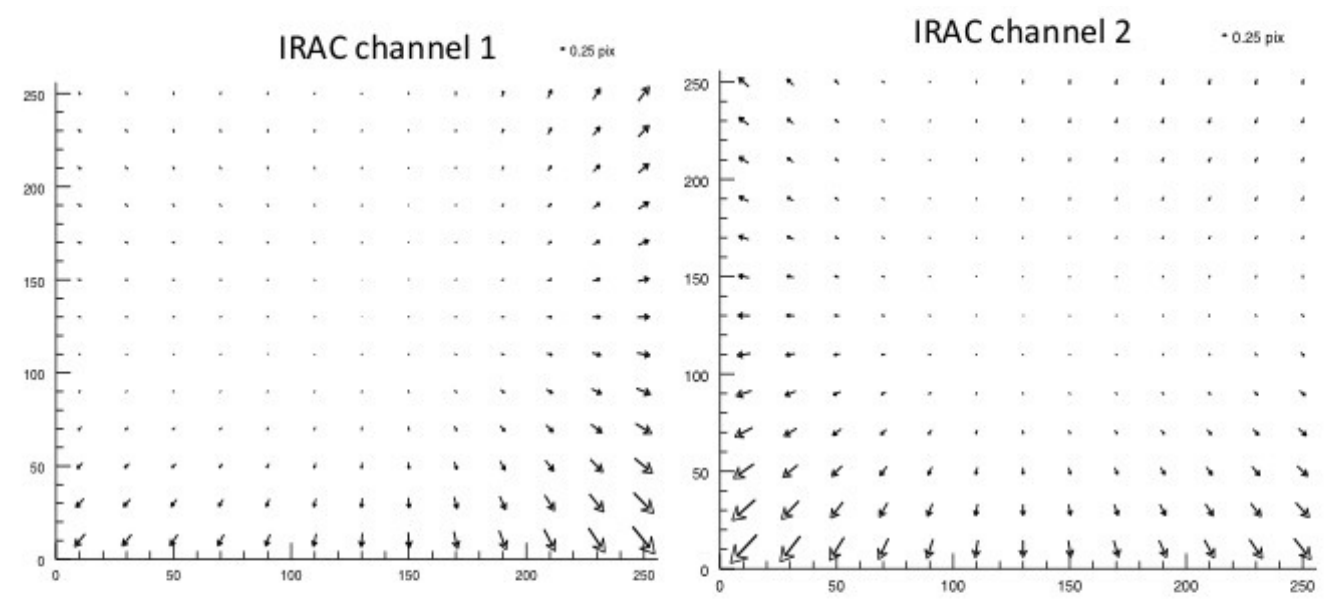

Scaling is $10 \mathrm{x}$

Figure 2. Developed and tested $5^{\text {th }}$ order solution using HST relative astrometry. The current accuracy improves to $\sim 20$ mas.

A least squares fitting was performed to fit the unaccounted distortion using various models, including the currently used Polynomial_SIP, and Polynomial2d, Chebyshev2d, and Legendre2d, without weighting. The difference between SIP Polynomial and Polynomial2d is that the SIP_Polynomial allows the combination of the exponents to equal the order and Polynomial2d allows the highest exponent to be the order. For example, a $3^{\text {rd }}$ order Polynominal2d allows $x^{1} y^{3}, x^{2} y^{3}$, etc while the SIP_Polynomial does not.
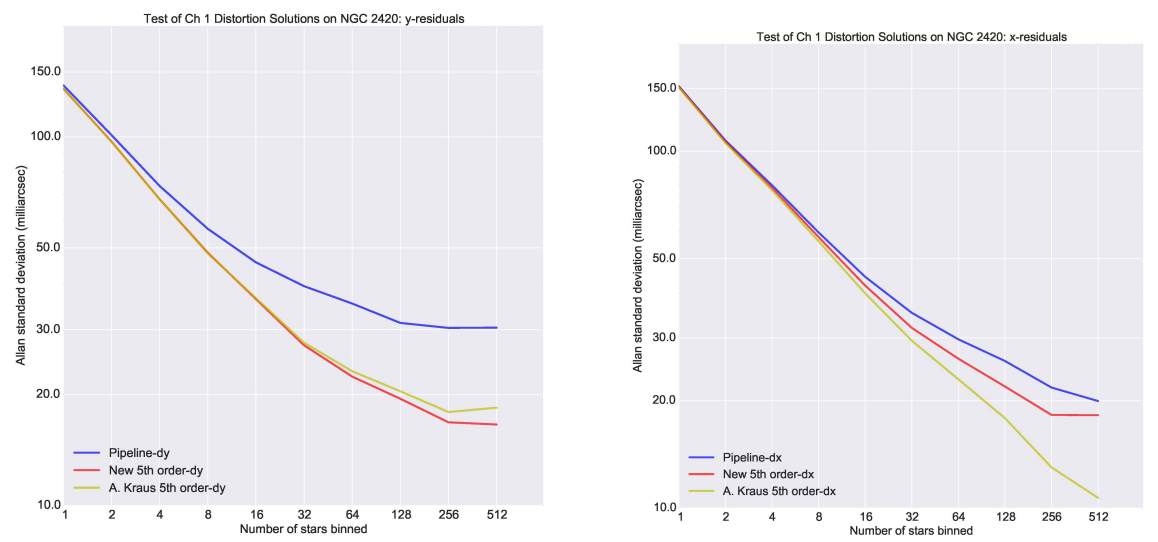

Figure 3. Allan variation testing of current distortion residuals. The current accuracy can improve to $\sim 20$ mas 
A $5^{\text {th }}$ order SIP_Polynomial solution was derived and found to be the best solution. It updated the pixel scale by $<1 \%$ and added an additional linear term to the polynomial. Data cleaning was excluded except for a couple of experiments, and training sets were used to check for model over-fitting. The differences between measured and "undistorted" positions using data combined with 10 -fold cross-validation, in which $90 \%$ of data is used as training and $10 \%$ as test, repeated 10 times. An Allan variance test was run on data using the new solution and residuals of $\sim 20$ mas could be reached with binning of multiple observations to reduce the residuals (Figure 3).

\subsection{Pointing Refinement catalog update}

Due to Spitzer blind pointing giving a rms of $\sim 0 . " 5$, a two step pointing refinement is run within the BCD pipeline. Point sources are identified and extracted from the pipeline processed products and correlated in position and flux to pointsource matches in the $2 \mathrm{MASS}^{11}$ point source catalog. For most stellar sources, intrinsic color will not cause large variations from the $\mathrm{K}$ band and IRAC bands. A minimum of five sources also excludes any bias due to individual sources. A new translational and rotational reference frame can then be computed using correlation differences in positions and uncertainties leading to a refinement of the pointing to be created. This typically improves the positional error to $<0 . " 3$ and removed any systematic offsets.

As the Spitzer observations are now occurring over 15 years after the 2MASS observations, the IRAC team understood there might be a significant effect of stellar proper motion upon the pointing refinement process. In examination of the UCAC4 catalog $^{12}$, it was found $22 \%$ of the 2 MASS stars bright enough to be used for the refinement process had proper motions. Further examination found the 2MASS catalog positional accuracy to be higher than the more recent ALLWISE $^{13}$ mission for stars observable within IRAC frame times most likely due to the larger beam size of the ALLWISE survey.

For Spitzer pointing refinement purposes, the 2MASS catalog was merged with the UCAC4 known proper motions. To verify these changes, observations from fields in the Bootes region that had been observed over five different epochs from the beginning of the mission in 2003 until 2014 were reprocessed and measured. Positions all agreed within measurement uncertainties and positional errors remain $<0 . " 3$ for data.

\section{CALIBRATION CHANGES}

\subsection{Flatfields}

Within the calibrated data pipeline, a flat-field map is used to account for the small pixel-to-pixel responsivity variation (i.e. DN/incident photon conversion). Due to the lack of a shutter, observations are taken of pre-selected regions of highzodiacal background. In the processing, all objects and outliers are detected and rejected, and the flat fields are normalized to one. Analysis during first years of the mission revealed the flat-field calibrations were static within the ability to measure. As a result, a "super-skyflat" was developed periodically.

For the warm mission, a "super-skyflat" had been created using the first two years of data. For the final reprocessing, all data have been processed with a new super skyflat created from over 5 years of data. The final precisions of the skyflats are $0.4 \%$ and $0.2 \%$, respectively for the channel 1 and 2 skyflats (Figure 4 ). 

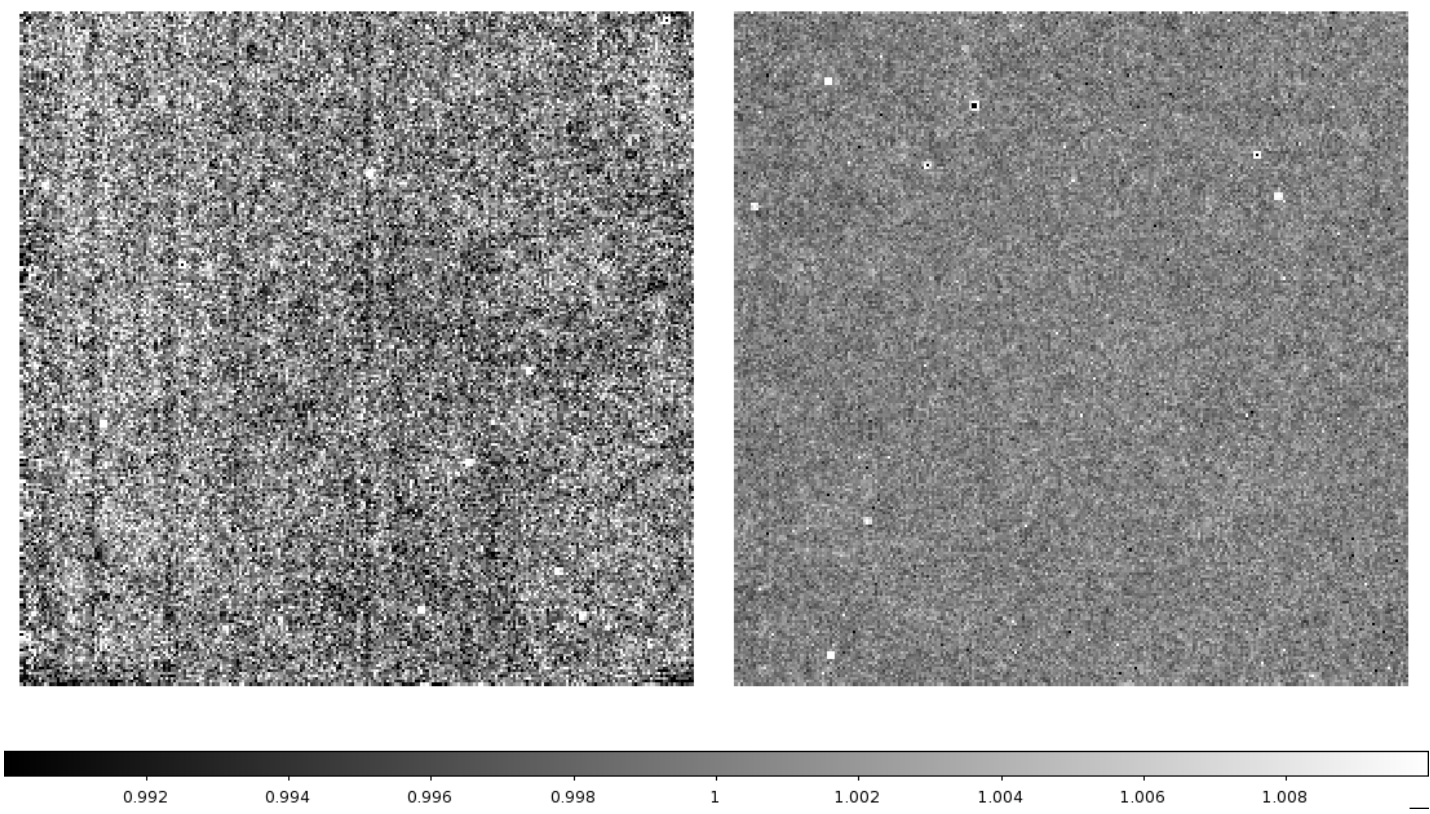

Figure 4. Ratio of the warm era superflats from just 2 years of data to 5 years of data. Final precisions are $0.4 \%$ and $0.2 \%$ for the channel 1 and 2.

\subsection{Linearity solution}

For previous versions of the pipeline, the linearity calibration has been derived using a combination of staring observations of bright galactic nebulae, and sources observed by the SWIRE ${ }^{14}$ Legacy science program during cryogenic operations. During the recent SERVS ${ }^{15}$ exploration science program, many of the same sources were re-observed. These observations were used to re-derive a more robust linearity solution. The final linearity solution currently applied in the processing includes additional adjustments incorporating the constancy of the PSF as a function of well depth. The new linearity solution should be accurate at the level of better than $0.5 \%$ up to above $90 \%$ full well (Figure 5).

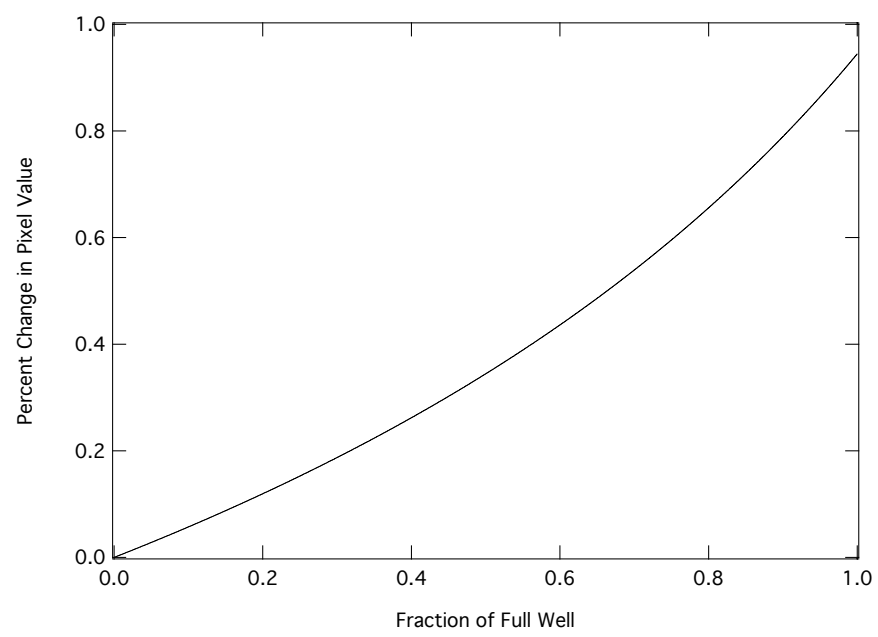

Figure 5. Change in linearized pixel intensity in channel 1 array as a $\%$ change vs fraction of full well. For pixel at full well, the change in flux from old pipeline version to final version is $<1 \%$ and is accurate to better than $0.5 \%$ for up to $90 \%$ full well. 


\subsection{Flux calibration}

The warm era flux calibration being used for the pipeline was derived previous to the final cryogenic flux calibration. ${ }^{16}$ It only had used the A stars and not the combination of the $\mathrm{K}$ standard stars. In this processing, as in the final cryogenic calibration, the flux calibration was derived using the combination 5 years of observations of $\mathrm{A}$ and $\mathrm{K}$ stars and updated spectral templates. ${ }^{17}$. The best derived pixel phase corrections and array-location dependent corrections were solved simultaneously for each measure and averaged over the entire array ${ }^{18}$. The overall flux calibration changed less than $0.5 \%$ in channel 1 and $1.49 \%$ in channel 2 , but agree better with independent tests.

\section{TIMING IS EVERYTHING}

\subsection{Nearest in time darks and exoplanet stares}

On IRAC, the darks are calculated using observations on an area of the sky absent of bright stars and galaxies. They are reduced similarly as science observations until after the fowler linearization step. At that point the images are medianed, removing all outliers and sources. Since the zodiacal light background covers the entire FOV, it is a background which can not be removed from the skydark. The background is removed from the science observation by subtraction of the skydark taken close in time to the observation, as the zodiacal background slowly varies throughout the year.

Due to this, dark observations in all exposure times are taken once a week and processed to be used for observations "nearest in time". However, this can cause some problems. The IRAC pipeline reduces observations frame by frame and calibrations are chosen for each frame. Due to the timing of an observation set, the beginning frames might receive one dark with which they are closest in time, while the remaining frames receive a second dark taken after the observation, but closer in time to the frames.

This problem coule manifest itself during a staring observation attempting to observe an eclipse of an exoplanet. If the dark changed, this could cause a small $(\sim 1 \%)$ offset in the bias of the frames from beginning to end, but large enough to cause an effect on detection of an eclipse (typically $<0.1 \%$ ). In answer to this, one dark needed to be "turned off" in the pipeline, and the data reprocessed. Thereby, the pipeline found one dark nearest in time to all frames and consistently used that one calibration file. After this was found, the pipeline monitored all staring mode observations for any change in calibration files and reprocessed before archiving.

\section{CONCLUSION}

Observations utilizing IRAC aboard the Spitzer Space Telescope will continue until mid-2019 based on the recent NASA senior review decision. Our final pipeline modifications have been finished and all warm mission data have been reprocessed as of July 2016. The project continues to have a goal of providing the best calibrated data for a wide range of science observations.

\section{REFERENCES}

[1] Fazio, G. G., Hora, J. L., Allen, L. E., Ashby, M. L. N., Barmby, P., Deutsch, L. K., Huang, J.-S., et al., "The Infrared Array Camera (IRAC) for the Spitzer Space Telescope", ApJS, 154, 10-17 (2004).

[2] Hora, J. L., Fazio, G. G., Allen, L. E., Ashby, M. L. N., Barmby, P., Deutsch, L. K., Huang, J.-S., et al., "In-flight performance and calibration of the Infrared Array Camera (IRAC) for the Spitzer Space Telescope", Proc. SPIE 5487, 77-92 (2004).

[3] Werner, M. N., Roellig, T. L., Low, F. J., Rieke, G. H., Rieke, M., Hoffmann, W. F., Young, E., et al., “The Spitzer Space Telescope Mission”, ApJS, 154, 1-9 (2004). 
[4] McMurtry, C. W., Pipher, J. L., Forrest, W. J., "Spitzer space telescope: dark current and total noise prediction for InSb detector arrays in the infrared array camera (IRAC) for the post-cryogen era", Proc. SPIE 6265, 08 (2006).

[5] Storrie-Lombardi, L. J., et al., "The Science Opportunities of the Warm Spitzer Mission”, AIP, 943 (2007).

[6] IRAC Instrument Handbook; http://irsa.ipac.caltech.edu/data/SPITZER/docs/irac/iracinstrumenthandbook/

[7] Perryman, M. A. C., de Boer, K. S., Gilmore, G., et al. A\&A, 369, 339 (2001)

[8] Kirkpatrick, J.D., et al ApJ 753156 (2012)

[9] Shupe, D.L., Moshir, M., Li, J., Makovoz, D., Narron, R., Hook, R.N. "The SIP Convention for Representing Distortion in FITS Image Header", ADASS XIV ASP Conference Series, Vol. 347, Proceedings of the Conference held 24-27 October, 2004 in Pasadena, California, USA. Edited by P. Shopbell, M. Britton, and R. Ebert. San Francisco: Astronomical Society of the Pacific, p.491, (2005)

[10] Stetson, P.B., Bruntt, H., Grundahl, F.; PASP 115413 (2003)

[11] Skrutskie, M.F. et al AJ 1311163 (2006)

[12] Zacharias,N., AJ 14544 (2013)

[13] Wright, E.L. et al AJ 1401868 (2010)

[14] Lonsdale, C.J., PASP 115897 (2003)

[15] Maudit, J.C. et al PASP 124714 (2012)

[16] Carey, S., et al; Space Telescopes and Instrumentation2012: Proceedings Vol 8442, 84421Z, 12 (2012)

[17] Engelke, C.W., Price, S.D, Kraemer ,K.E.; AJ 132, 1445 (2006)

[18] Carey, S., et al; Space Telescopes and Instrumentation2016: this issue 